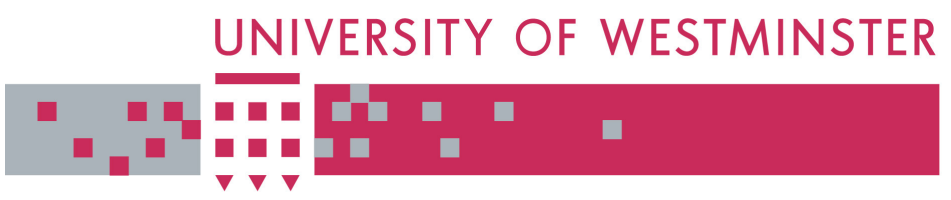

WestminsterResearch

http://www.wmin.ac.uk/westminsterresearch

\title{
Correction of transmitter gain and phase errors at the receiver.
}

Ediz Cetin

Izzet Kale

Richard Morling

Cavendish School of Computer Science

Copyright (C [2002] IEEE. Reprinted from IEEE International Symposium on Circuits and Systems (ISCAS 2002), 26-29 May 2002, Arizona, USA.

This material is posted here with permission of the IEEE. Such permission of the IEEE does not in any way imply IEEE endorsement of any of the University of Westminster's products or services. Internal or personal use of this material is permitted. However, permission to reprint/republish this material for advertising or promotional purposes or for creating new collective works for resale or redistribution must be obtained from the IEEE by writing to pubs-permissions@ieee.org. By choosing to view this document, you agree to all provisions of the copyright laws protecting it.

The WestminsterResearch online digital archive at the University of Westminster aims to make the research output of the University available to a wider audience. Copyright and Moral Rights remain with the authors and/or copyright owners. Users are permitted to download and/or print one copy for non-commercial private study or research. Further distribution and any use of material from within this archive for profit-making enterprises or for commercial gain is strictly forbidden.

Whilst further distribution of specific materials from within this archive is forbidden, you may freely distribute the URL of WestminsterResearch.

(http://www.wmin.ac.uk/westminsterresearch).

In case of abuse or copyright appearing without permission e-mail wattsn@wmin.ac.uk. 


\title{
CORRECTION OF TRANSMITTER GAIN AND PHASE ERRORS AT THE RECEIVER
}

\author{
Ediz Çetin, Izzet Kale and Richard C. S. Morling \\ University of Westminster, Department of Electronic Systems, \\ Applied DSP and VLSI Research Group, \\ London W1W 6UW, United Kingdom
}

\begin{abstract}
This paper explores the benefits of compensating transmitter gain and phase imbalances in the receiver for quadrature communication systems. It is assumed that the gain and phase imbalances are introduced at the transmitter only. A simple nondata aided DSP algorithm is used at the receiver to compensate for the imbalances. Computer simulation has been performed to study a coherent QPSK communication system.
\end{abstract}

\section{INTRODUCTION}

Gain and phase imbalances in quadrature transmitters are known to degrade the overall communication link performance [1]-[3]. Transmitters with good balance in the in-phase and quadrature channels must rely on stringent specifications on components that are difficult to achieve as the frequency of operation increases to microwave or $\mathrm{mm}$-wave region, especially in direct transmitter designs.

Several techniques have been proposed to estimate and compensate the quadrature modulator imperfections at the transmitter [4]-[7]. Many of these approaches imply the use of analog feedback loop around the quadrature modulator. This in itself is not an error free loop. In [8] the Gram-Schmidt orthogonalization procedure is proposed for correcting the transmitter errors at the receiver by using test signals. This paper explores the capability of a non-data aided adaptive DSP technique developed for quadrature receivers in [9] to compensate for the imbalances introduced at the transmitter. Indeed, the analysis carried out in [9] assumed an ideal transmitter where the imbalances were introduced only at the receiver. In this study, we assume that the gain and phase imbalances are introduced at the transmitter only and examine the effects of compensating them in the receiver.

The paper is organized as follows: Section 2 defines the model of the gain and phase imbalances in the transmitter. Section 3 describes how the transmitter gain and phase imbalances are compensated at the receiver, while simulation results and concluding remarks are given in Sections 4 and 5 respectively.

\section{MODELLING THE TRANSMITTER GAIN AND PHASE IMBALANCES}

Ideally, the in-phase (I) and quadrature (Q) channels of a quadrature communication system are orthogonal to each other. However, due to implementation imperfections, there always exist gain and phase imbalances that destroy the orthogonality between these two channels and degrade the performance of the communication system. [1]-[9]

The gain and phase modelling is a complex issue. To facilitate analysis, we use the quadrature transmitter model in Figure 1 and assume that phase and gain errors are differentially distributed between the I and $Q$ channels.

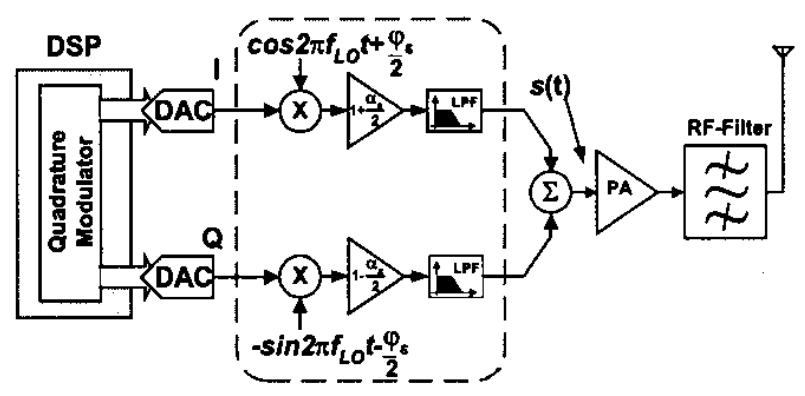

Figure 1 Typical quadrature transmitter

Carrier modulated signal $s(t)$ at the output of the ideal analog quadrature modulator is given as:

$$
\begin{aligned}
s(t) & =\mathfrak{R}\left[u(t) e^{j 2 \pi f_{R F^{t}}}\right] \\
& =u_{I}(t) \cos \left(2 \pi f_{R F} t\right)-u_{Q}(t) \sin \left(2 \pi f_{R F} t\right)
\end{aligned}
$$

where $u_{N}(\mathrm{t})$ and $u_{Q}(\mathrm{t})$ are the baseband in-phase and quadrature components, each having unity power, $2 \pi f_{R F} t$ is the carrier frequency. The complex envelope, $u(t)$ of the RF signal $s(t)$ can be expressed as a 1-by-2 column vector containing the baseband in-phase and quadrature components. Hence the $u(t)$ can be represented as:

$$
u(t)=\left[u_{I}(t), u_{Q}(t)\right]^{T} \quad \text { where } \begin{array}{r}
u_{l}(t)=a(t) \cos (\phi(t)) \\
u_{Q}(t)=a(t) \sin (\phi(t))
\end{array}
$$

where $a(t)$ is the envelope, and $\phi(t)$ is the phase. In the presence of phase and gain mismatches equation (1) becomes:

$$
\begin{gathered}
s(t)=u_{I}(t)\left[1+0.5 \alpha_{\varepsilon}\right] \cos \left(2 \pi f_{R F} t+0.5 \varphi_{\varepsilon}\right)- \\
u_{Q}(t)\left[1-0.5 \alpha_{\varepsilon}\right] \sin \left(2 \pi f_{R F} t-0.5 \varphi_{\varepsilon}\right)
\end{gathered}
$$

where $\varphi_{\varepsilon}$ is the phase and $\alpha_{\varepsilon}$ is the gain error. Equation (3) can be expressed as

$$
\begin{aligned}
s(t)= & \cos \left(2 \pi f_{R F} t\right)\left[u_{I}(t) g_{1} \cos 0.5 \varphi_{\varepsilon}+u_{Q}(t) g_{2} \sin 0.5 \varphi_{\varepsilon}\right]- \\
& \sin \left(2 \pi f_{R F} t\right)\left[u_{I}(t) g_{1} \sin 0.5 \varphi_{\varepsilon}+u_{Q}(t) g_{2} \cos 0.5 \varphi_{\varepsilon}\right]
\end{aligned}
$$


where $g_{1}=\left(1+0.5 \alpha_{\varepsilon}\right)$ and $g_{2}=\left(1-0.5 \alpha_{\varepsilon}\right)$. To gain more insight into the effects of analog modulator mismatches, suppose the RF signal $s(\mathrm{t})$ is QPSK modulated. Figure 2 shows the effect of these mismatches on the signal constellation.

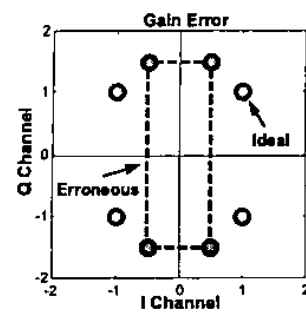

(a)

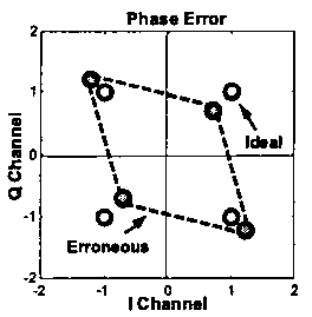

(b)
Figure 2 QPSK constellation with (a) Gain, (b) Phase imbalance (gain error of $6 \mathrm{~dB}$ and phase error of $15^{\circ}$ )

The effect of phase and gain mismatches can also be seen by examining the QPSK signals in the time domain as shown in Figure 3. The gain error simply appears as a non-unity scale factor in the amplitude. The phase imbalance, on the other hand, corrupts each channel by a fraction of the data pulses in the other channel, in essence degrading the signal-to-noise ratio.
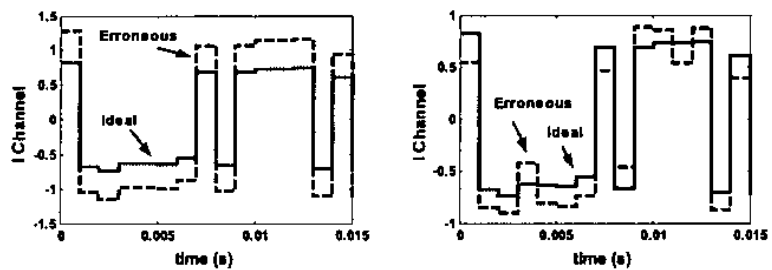

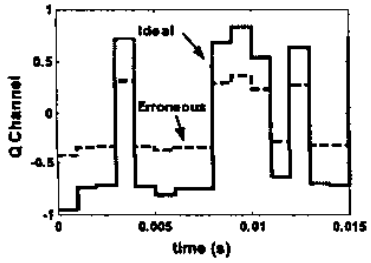

(a)

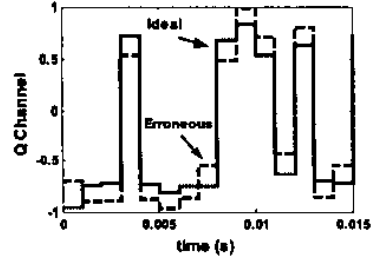

(b)
Figure 3 Effect of V/Q mismatch on QPSK waveform with (a) Gain, (b) Phase imbalance (gain error of $6 \mathrm{~dB}$ and phase error of $15^{\circ}$ )

As it can be seen from Figure 2, the phase error rotates the quadrature axis distorting the quadrature modulator's coordinate system. Gain mismatch on the other hand results in a rectangle instead of a square. It can be seen that each transmitted symbol undergoes a transformation, or mapping onto the transmitted signal constellation. This transformation can be expressed as:

$$
s(t)=\mathbf{M}\{u(t)\}
$$

where:

$$
\mathbf{M}= \begin{cases}{\left[\begin{array}{ll}
\left(1+0.5 \alpha_{\varepsilon}\right) \cos \left(\varphi_{\varepsilon} / 2\right) & \left(1-0.5 \alpha_{\varepsilon}\right) \sin \left(\varphi_{\varepsilon} / 2\right) \\
\left(1+0.5 \alpha_{\varepsilon}\right) \sin \left(\varphi_{\varepsilon} / 2\right) & \left(1-0.5 \alpha_{\varepsilon}\right) \cos \left(\varphi_{\varepsilon} / 2\right)
\end{array}\right] \quad \text { ideal }} \\
\text { erronous }\end{cases}
$$

Another effect of the analog quadrature modulator errors is the generation of a spurious signal. In the presence of quadrature errors equation (1) can be re-written as:

$$
\begin{aligned}
& s(t)=\frac{1}{2} \mathfrak{R}\left\{u(t)[\overbrace{e^{j\left(2 \pi f_{R f} t\right)}}^{\text {Desired }}\left(g_{1} e^{j \frac{\Phi_{\varepsilon}}{2}}-g_{2} e^{-j \frac{\Phi_{k}}{2}}\right)+\right. \\
& \underbrace{\left.e^{-j\left(2 \pi f_{R F} t\right)}\left(g_{1} e^{-j \frac{\Phi_{\varepsilon}}{2}}+g_{2} e^{j \frac{\Phi_{\varepsilon}}{2}}\right)\right]}_{\text {Spurious }}\}
\end{aligned}
$$

where $g_{1}=\left(1+0.5 \alpha_{\varepsilon}\right)$ and $g_{2}=\left(1-0.5 \alpha_{\varepsilon}\right)$. From equation (7) the corresponding amplitudes of the desired and spurious signals are

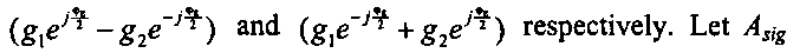
denote the amplitude of the desired signal and $A_{s p r}$ the amplitude of the spurious signal. The ratio between them is the sideband suppression. As a function of phase and gain errors the sideband suppression can be expressed in decibels as:

$$
\begin{aligned}
& 10 \log \left(\frac{A_{s p r}}{A_{s i g}}\right)^{2}=10 \log \left(\frac{2-2 \cos \varphi_{\varepsilon}+0.5 \alpha_{\varepsilon}{ }^{2}\left(1+\cos \varphi_{\varepsilon}\right)}{2+2 \cos \varphi_{\varepsilon}+0.5 \alpha_{\varepsilon}{ }^{2}\left(1-\cos \varphi_{\varepsilon}\right)}\right) \\
& \text { Pheo Eror (dog) }
\end{aligned}
$$

Figure 4 Single Sideband Suppression as a function of $\alpha_{\varepsilon}$ and $\varphi_{\varepsilon}$

\section{BLIND TRANSMITTER GAIN AND PHASE IMBALANCE CORRECTION}

The received and quadrature downconverted baseband signal $r(t)$ at the receiver can be expressed as:

$$
r(t)=s(t)+n(t)
$$

where $s(\mathrm{t})$ is the erroneously generated transmitted signal and $n(t)$ is the complex-valued additive white Gaussian noise. In the ideal case where there are no transmitter phase and gain mismatches the received in-phase, $r_{I}$ and quadrature, $r_{Q}$ signals relate to the transmitted quadrature components $u_{l}$ and $u_{Q}$ as:

$$
\left[\begin{array}{l}
r_{I}(t) \\
r_{Q}(t)
\end{array}\right]=\underbrace{\left[\begin{array}{ll}
1 & 0 \\
0 & 1
\end{array}\right]}_{M} \times\left[\begin{array}{l}
u_{I}(t) \\
u_{Q}(t)
\end{array}\right]
$$

where $M$ is the mixing matrix which transforms the $u_{\mathrm{I}}(\mathrm{t})$ and $u_{\mathrm{Q}}(\mathrm{t})$ signals to the new $r_{1}(\mathrm{t})$ and $r_{\mathrm{Q}}(\mathrm{t})$ signals. Hence, in the ideal case $M=I$, as shown in equation (10). Also, it can be seen that in the ideal case there is no crosstalk between the I and Q channels and they are orthogonal. However, in the presence phase and gain errors this no longer holds and the received signal components relate to the transmitted ones as: 


$$
\left[\begin{array}{l}
r_{l}(t) \\
r_{Q}(t)
\end{array}\right]=\underbrace{\left[\begin{array}{ll}
\left(1+0.5 \alpha_{\varepsilon}\right) \cos \left(\varphi_{\varepsilon} / 2\right) & \left(1-0.5 \alpha_{\varepsilon}\right) \sin \left(\varphi_{\varepsilon} / 2\right) \\
\left(1+0.5 \alpha_{\varepsilon}\right) \sin \left(\varphi_{\varepsilon} / 2\right) & \left(1-0.5 \alpha_{\varepsilon}\right) \cos \left(\varphi_{\varepsilon} / 2\right)
\end{array}\right]}_{M} \times\left[\begin{array}{l}
u_{I}(t) \\
u_{Q}(t)
\end{array}\right]
$$

As it can be seen from (11), due to the phase and gain mismatches the quadrature components are no longer uncorrelated and orthogonal. The effect of transmitter I and $Q$ channel phase and gain mismatches on the Bit-Error-Rate (BER) performance of the quadrature receiver is shown in Figure 5.
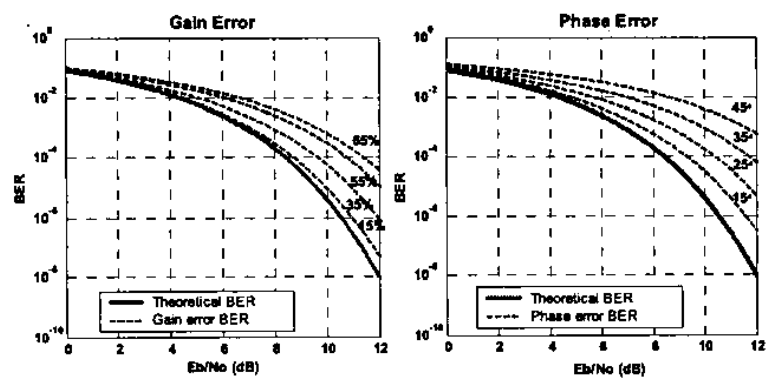

Figure 5 BER as a function of phase and gain ertors

The simplified model for the proposed adaptive source separation approach is depicted in Figure 6.

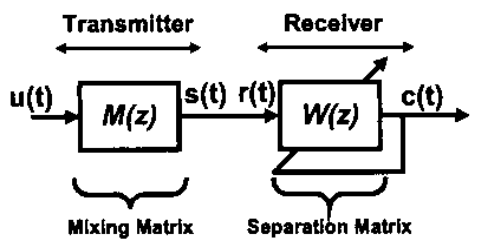

Figure 6. Source separation approach

The mixing matrix $M(z)$ of equation (11) can be simplified as:

$$
M(z)=\left[\begin{array}{cc}
1 & M_{Q}(z) \\
M_{I}(z) & 1
\end{array}\right]
$$

where $M_{Q}(\mathrm{z})$ determines the crosstalk from the $u_{\mathrm{Q}}(\mathrm{t})$ to $r_{\mathrm{I}}(\mathrm{t})$ and $M_{I}(\mathrm{z})$ determines the crosstalk from the $u_{\mathrm{I}}(\mathrm{t})$ to $r_{\mathrm{Q}}(\mathrm{t})$ channels. Both $M_{\Lambda}(\mathrm{z})$ and $M_{Q}(\mathrm{z})$ depend on the amount of the transmitter phase and gain errors. The proposed feed-forward blind decorrelator along with the assumed phase and gain mismatch model is shown in Figure 7.

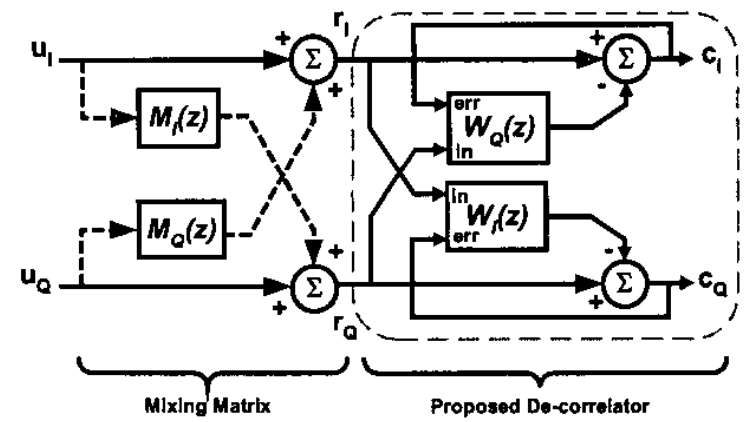

Figure 7. Proposed solution.

In the proposed approach, both $W_{f}(\mathrm{z})$ and $W_{Q}(\mathrm{z})$ are two tap LMS based adaptive FIR filters arranged in an Adaptive Noise Canceller (ANC) set-up. These FIR filters, $W_{/}(\mathrm{z})$ and $W_{Q}(\mathrm{z})$, operate on the baseband signal and try to model $M_{\mathcal{O}}(\mathrm{z})$ and $M_{\boldsymbol{f}}(\mathrm{z})$. Hence doing so they de-correlate the two signals, $r_{1}$ and $r_{\mathrm{Q}}$. The transfer matrix, $T(\mathrm{z})$, from the sources $u_{\mathrm{I}}$ and $u_{\mathrm{Q}}$ to the source estimates $c_{\mathrm{I}}$ and $c_{\mathrm{Q}}$ is given as:

$$
\begin{aligned}
\mathbf{T}(z) & =\mathbf{W M} \\
& =\left[\begin{array}{cc}
1 & -W_{I}(z) \\
-W_{Q}(z) & 1
\end{array}\right]\left[\begin{array}{cc}
1 & M_{I}(z) \\
M_{Q}(z) & 1
\end{array}\right]
\end{aligned}
$$

This equation can be solved for $c_{\mathrm{I}}$ and $c_{\mathrm{Q}}$ to give:

$$
\left[\begin{array}{l}
c_{I} \\
c_{Q}
\end{array}\right]=\left[\begin{array}{cc}
1 & -W_{I}(z) \\
-W_{Q}(z) & 1
\end{array}\right] \times M(z) \times\left[\begin{array}{l}
u_{I} \\
u_{Q}
\end{array}\right]
$$

Based on the orthogonal principle, the filter $W_{\Lambda}(z)$ decorrelates its error signal $c_{\mathrm{Q}}$ with the input signal $r_{\mathrm{l}}$; while the filter $W_{Q}(\mathrm{z})$ decorrelates its input signal $r_{Q}$ with the error signal $c_{\mathrm{l}}$. Thus, the output signals $c_{\mathrm{I}}$ and $c_{\mathrm{Q}}$ are statistically uncorrelated, that is,

$$
E\left[c_{I}(n) \times c_{Q}(n-k)\right]=0, \quad \forall k,
$$

From (13) and (14), it is clear that the choice of the following solution:

$$
\left[\begin{array}{l}
W_{I}(z) \\
W_{Q}(z)
\end{array}\right]=\left[\begin{array}{l}
M_{l}(z) \\
M_{Q}(z)
\end{array}\right]
$$

yields:

$$
\left[\begin{array}{l}
c_{I}(n) \\
c_{Q}(n)
\end{array}\right]=\alpha \times\left[\begin{array}{l}
u_{I}(n) \\
u_{Q}(n)
\end{array}\right]
$$

where, $\alpha=1-M_{I}(z) M_{Q}(z)$. Hence, $W_{I}(z)$ models $M_{N}(z)$ and $W_{Q}(\mathrm{z})$ models $M_{Q}(\mathrm{z})$. These estimates are then subtracted eliminating the crosstalk between the two channels. In doing so the two channels are de-correlated.

\section{SIMULATION RESULTS}

To verify the analysis for the transmitter gain and phase imbalances and to study the effect of compensating the transmitter gain and phase imbalance at the receiver, a coherent QPSK system has been simulated. To focus on the effects of the gain and phase imbalance compensation, we assume that the carrier and the symbol timing are perfectly synchronized. We consider two cases; one with phase error of $15^{\circ}$ and gain error of $5 \mathrm{~dB}$ and the other case with phase error of $30^{\circ}$ and gain error of $5 \mathrm{~dB}$. The channel is assumed to be a AWGN channel with variance of 0.01 . Constellation and eye diagrams for the first case are shown in Figures 8 and 9. As it can be seen from Figures 8(b) and 9(b), the transmitter phase and gain errors have distorted the constellation and eye diagrams. As is clear from Figure 8(b) the effect of the phase and gain error is to rotate and scale the projection of the received signal in the signal space getting closer to the edge of its decision region. As a result, it takes less noise power to perturb the projection and move it into the wrong decision region. The result is a higher probability of bit error rate than would otherwise be expected. They eye diagram of Figure 9 (b) is almost completely closed due to the phase and gain errors. It is very difficult to make a sensible decision about the data sent. Figures $8(\mathrm{c})$ and 9 (c) depict the output of our adaptive algorithm. 
The constellation diagram of Figure $8(\mathrm{~b})$ is de-rotated back almost matching the ideal (a). The eye diagram of Figure 9(c) opened the erroneous case (b) substantially almost matching the ideal (a).

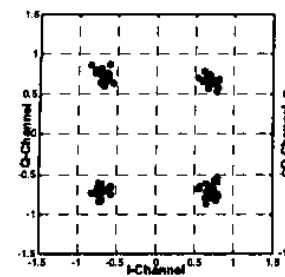

(a) Ideal

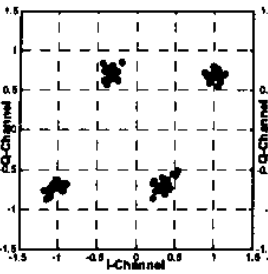

(b) Erroneous

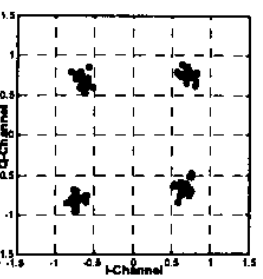

(c) Corrected
Figure 8 Constellation diagrams for (a) Ideal, (b) Erroneous and

(c) Corrected, for $15^{\circ}$ and $5 \mathrm{~dB}$ gain and phase error

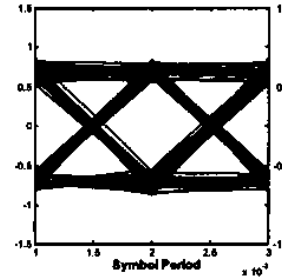

(a) Ideal

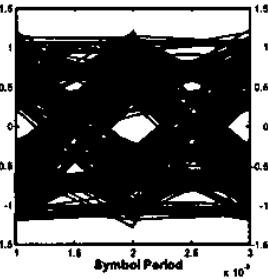

(b) Erroneous

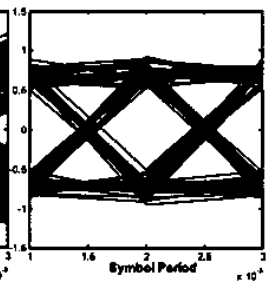

(c) Corrected
Figure 9. Eye diagrams for (a) Ideal, (b) Erroneous and (c) Corrected, for $15^{\circ}$ and $5 \mathrm{~dB}$ gain error.

Figures 10 and 11 depict the constellation and eye diagrams for the second case. Our adaptive algorithm is applied to the erroneous constellation Figure $10(\mathrm{~b})$ to yield (c). This is a much better performance in comparison to (b) and closely tracks the ideal in (a). They eye diagram of Figure 11(b) is closed and no sensible decision about the data sent can be made. Figures 10(c) and 11(c) depict the output of our adaptive algorithm. The constellation diagram of Figure $10(\mathrm{~b})$ is de-rotated back to almost match the ideal (a) and the eye diagram of Figure 11(b) is substantially opened as in (c) almost matching the ideal of (a).

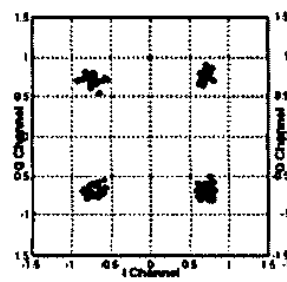

(a) Ideal

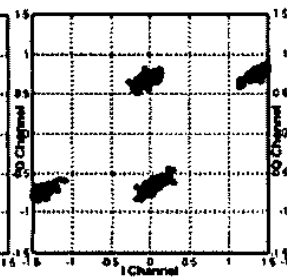

(b) Erroneous

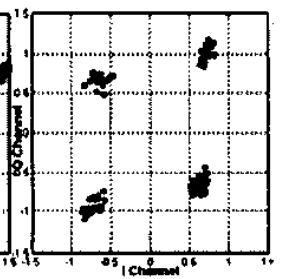

(c) Corrected
Figure 10 Constellation diagrams for (a) Ideal, (b) Erroneous and (c) Corrected, for $30^{\circ}$ and $5 \mathrm{~dB}$ gain and phase error

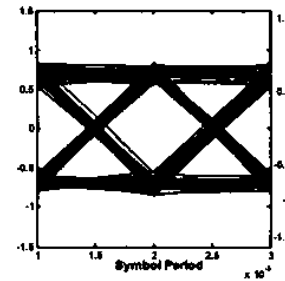

(a) Ideal

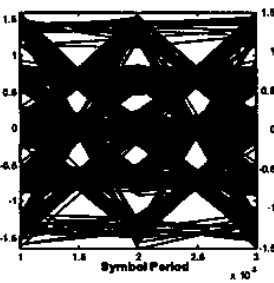

(b) Erroneous

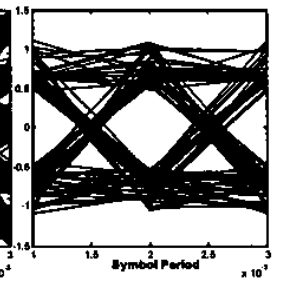

(c) Corrected
Figure 11. Eye diagrams for (a) Ideal, (b) Erroneous and (c) Corrected, for $30^{\circ}$ and $5 \mathrm{~dB}$ gain error.

\section{CONCLUDING REMARKS}

This paper studied the potential advantages of compensating transmitter gain and phase imbalances in the receiver for quadrature communication systems. A gain and phase model has been defined to facilitate the study. A simple blind LMS-based adaptive algorithm using two, 2-tap adaptive FIR filters are employed at the receiver to compensate the transmitter gain and phase imbalances. Computer simulations have been carried out to understand and demonstrate the performance of the adaptive compensation scheme.

\section{REFERENCES}

[1] J. K. Cavers and M. W. Liao, "Adaptive compensation for imbalance and offset losses in direct conversion transceivers," IEEE Trans. Veh. Technol, vol. 42, no. 4, pp. 581-588, Nov 1993.

[2] National Semiconductor Application note 899, "Operating and Evaluating Quadrature Modulator for Personal Communication Systems ", October 1993.

[3] A.E. Jones , T.A.H. Wilkinson and J.G. Gardiner, "Effects of modulator deficiencies and amplifier nonlinearities on the phase accuracy of GMSK signalling", IEE Proceedings I on Communications, Speech and Vision, vol. 140, Issue: 2 , pp.157-162, April 1993.

[4] S.A. Leyonhjelm and M. Faulkner, "Digital signal processing and direct conversion for multichannel transmitters", Vehicular Technology Conference, vol.1, pp. $494-498,1994$.

[5] S.O. Ampem-Darkko and H.S Al-Raweshidy, "A novel technique for gain/phase error cancellation in LINC transmitters". Vehicular Technology Conference (VTC 1999 - Fall), vol. 4, pp. $2034-2038,1999$.

[6] R. Marchesani, "Digital precompensation of imperfections in quadrature modulators", IEEE Transactions on Communications, vol. 48, Issue: 4, pp. $552-556$, April 2000.

[7] M. Faulkner, T. Mattsson and W. Yates, "Automatic adjustment of quadrature modulators"; Electronics Letters , vol. 27 Issue: 3 , pp. $214-216,31$ Jan. 1991.

[8] X. Huang, "On transmitter gain/phase imbalance compensation at receiver" IEEE Communications Letters, vol. 4, Issue: 11, pp. $363-365$, Nov. 2000.

[9] Cetin, E., I. Kale and R. C. S. Morling, "Adaptive Compensation of Analog Front-end I/Q Mismatches in Digital Receivers", International Symposium on Circuits and Systems, (ISCAS 2001), Sydney, Australia, 6-9 May 2001 . 